Hagedoor, P., Groenewegen, P.P., Roberts, H., Helbich, M. Is suicide mortality associated with neighbourhood social fragmentation and deprivation? A Dutch register-based case-control study using individualised neighbourhoods. Journal of Epidemiology \& Community Health: 2020, 74(2), p. 197-202

$\begin{array}{lll}\text { Postprint version } & : & 1.0 \\ \text { Journal website } & : & \text { https://jech.bmj.com/content/74/2/197 } \\ \text { Pubmed link } & : & \text { https://www.ncbi.nlm.nih.gov/pubmed/31727790 } \\ \text { DOI } & : & \text { https://doi.org/10.1136/jech-2019-212699 }\end{array}$

This is a Nivel certified Post Print, more info at nivel.nl

\title{
Is suicide mortality associated with neighbourhood social fragmentation and deprivation? A Dutch register-based case-control study using individualised neighbourhoods
}

\author{
Paulien Hagedoorn ${ }^{1}$, Peter P Groenewegen, ${ }^{1,2}$ Hannah Roberts, ${ }^{1}$ Marco \\ Helbich $^{1}$
}

1 Department of Human Geography and Spatial Planning, Utrecht University, Utrecht, The Netherlands

2 Netherlands Institute for Health Services Research, Utrecht, The Netherlands

\begin{abstract}
ABSRACT
Background Neighbourhood social fragmentation and socioeconomic deprivation seem to be associated with suicide mortality. However, results are inconclusive, which might be because dynamics in the social context are not well-represented by administratively bounded neighbourhoods at baseline. We used individualised neighbourhoods to examine associations between suicide mortality, social fragmentation, and deprivation for the total population as well as by sex and age group.

Methods Using a nested case-control design, all suicides aged 18-64 years between 2007 and 2016 were selected from longitudinal Dutch register data and matched with 10 random controls. Indices for social fragmentation and deprivation were calculated annually for 300, 600 and 1000 metre circular buffers around each subject's residential address.

Results Suicide mortality was significantly higher in neighbourhoods with high deprivation and social fragmentation. Accounting for individual characteristics largely attenuated these associations. Suicide mortality remained significantly higher for women living in highly fragmented neighbourhoods in the fully adjusted model. Agestratified analyses indicate associations with neighbourhood fragmentation among women in older age groups (40-64 years) only. Among men, suicide risk was lower in fragmented neighbourhoods for those aged 18-39 years and for short-term residents. In deprived neighbourhoods, suicide risk was lower for men aged 40-64 years and long-
\end{abstract}


Hagedoor, P., Groenewegen, P.P., Roberts, H., Helbich, M. Is suicide mortality associated with neighbourhood social fragmentation and deprivation? A Dutch register-based case-control study using individualised neighbourhoods. Journal of Epidemiology \& Community Health: 2020, 74(2), p. 197-202

term residents. Associations between neighbourhood characteristics and suicide mortality were comparable across buffer sizes.

Conclusion Our findings suggest that next to individual characteristics, the social and economic context within which people live may both enhance and buffer the risk of suicide.

\section{Introduction}

Being the fifth leading cause of death among middle-aged adults worldwide, ${ }^{1}$ suicide remains a key public health issue. As evidence that neighbourhood characteristics correlate with mental health outcomes is mounting, ${ }^{2}$ there is growing interest in the influence of the social living environment on suicide mortality. ${ }^{3}$

Previous research showed positive associations between area-level socioeconomic disadvantages and suicidal behaviour. ${ }^{45}$ However, most of these studies relied on cross-sectional, ecological research designs that are unable to determine whether such associations were a consequence of spatial clustering of high-risk individuals (ie, composition) or whether they were the outcome of conditions of the living environment (ie, context). After accounting for individual socioeconomic characteristics, European multilevel studies reported mixed findings. Some studies reported an increased suicide risk in neighbourhoods with high deprivation and low social cohesion, ${ }^{67}$ while others found no correlation between neighbourhood socioeconomic characteristics and suicide mortality. ${ }^{8-10}$ These contradictory findings may be partly attributed to the definition of the spatiotemporal context that people belong to. ${ }^{11}$ Studies relied on socioeconomic characteristics of administratively bounded neighbourhoods measured at baseline. ${ }^{45}$ Such a conceptualisation fails to incorporate temporal dynamics in the social context; partly as a result of residential and social mobility. ${ }^{12}$ Moreover, administrative areas are prone to several methodological limitations (see Helbich, 201813 for a discussion). Many of these limitations can be overcome by using individualised neighbourhoods across space and time which reflect dynamic exposures to an individual's local living environment more accurately. ${ }^{14}$ However, to our knowledge, no study on suicide has implemented such an operationalisation of the neighbourhood social context.

In light of these shortcomings, this study employed longitudinal register data during a 10-year

follow-up period georeferenced at the address level to examine how the socioeconomic context affects suicide risk in the Netherlands. Although the Dutch suicide rate in 2007 was relatively low compared with other European countries ( 8 per 100000 persons), rates have been steadily increasing over time. ${ }^{15}$ This study aimed to 1 ) assess whether local social fragmentation and socioeconomic deprivation is associated with suicide mortality among adults aged 18-64 years and 2) assess this association by sex and age group as previous research indicated that neighbourhood characteristics might affect suicide mortality differently depending on sex and age. ${ }^{816}$

\section{Methods}

\section{Study design and suicide data}

Detailed information on the data and studydesign can be found elsewhere. ${ }^{17}$ We used apopulationbased nested case-control design. Longitudinal register data from 1 January 2007 until 31 December 2016 were obtained from the System of Social Statistical Datasets, maintained by Statistics Netherlands. ${ }^{18}$ Cases and controls were selected from the non-institutionalised Dutch population living in the Netherlands $\geq 10$ years. We identified all persons aged 18-64 years during 2007-2016 who died from suicide (ICD-10 codes $X 60-X 84$ ) as cases ( $n=10954)$. Cases with incomplete residential histories or missing individual or neighbourhood data $(n=1043)$ were excluded. For each case, we selected a random sample of 10 controls with the same age and sex profile who were alive at the 
Hagedoor, P., Groenewegen, P.P., Roberts, H., Helbich, M. Is suicide mortality associated with neighbourhood social fragmentation and deprivation? A Dutch register-based case-control study using individualised neighbourhoods. Journal of Epidemiology \& Community Health: 2020, 74(2), p. 197-202

date of suicide (matching date) by using incidence-density sampling and matching on year of birth, seks and calendar time. By employing this sampling procedure, the odds ratio (OR) from a casecontrol study approximates the rate ratio in the full population. ${ }^{19}$ After the sampling, 461 controls with missing neighbourhood characteristics were excluded and not replaced. The final study population consisted of 108560 individuals; 9911 cases and 98649 controls.

\section{Neighbourhood characteristics}

Neighbourhood socioeconomic characteristics were measured by individualised neighbourhoods. Using georeferenced addresses from the land registry, we centred circular buffers on the residential locations of each case and control. As previous research has shown that associations between neighbourhood characteristics and mental health might be scale dependent, ${ }^{20}$ we considered buffers with radii of 300,600 and $1000 \mathrm{~m}$. For comparison, we also used the administrative neighbourhood ('buurt'), the most detailed territorial unit in the Netherlands.

We computed social fragmentation, socioeconomic deprivation and urbanicity annually for 2007 until 2016 by aggregating individual characteristics of all residents living within a buffer (or neighbourhood) at 1 January of each year. The social fragmentation index, reflecting low levels of community integration, was based on the percentage of adult residents ( $>18$ years) who are unmarried, live in a single-person household, and who moved to the address in the last year. ${ }^{21}$ The deprivation index was calculated by the unemployment rate, the standardised median household income (reverse coded) and the share of households with a standardised income below the poverty line for the population at 1 January. To construct both indices, each input variable was $z$-scored and summed, with higher scores referring to higher levels of social fragmentation and deprivation. To control for urban-rural inequalities in suicide mortality, ${ }^{22}$ urbanicity was included. The indicator was operationalised, as advised elsewhere, ${ }^{23}$ through population density within each buffer (or neighbourhood) at 1 January of each year.

For each subject, we selected the social fragmentation index, deprivation index and urbanicity for the residential address and year corresponding to the matching date to reflect the sociospatial context at time of suicide. To facilitate comparisons with previous studies, ${ }^{68}$ each indicator was divided into quartiles based on the number of subjects.

\section{Individual characteristics}

Besides sex and age, already controlled for in the matched case-control design, ${ }^{24}$ we adjusted for several individual characteristics related to suicide risk. ${ }^{1625}$ As life events shortly before deaths may have triggered suicide, ${ }^{26}$ we considered individual characteristics around matching time. The following characteristics were obtained from the population register at the matching date: ethnic origin (Dutch or other), marital status (married, never married or not currently married), household type (couple with kids, couple without kids, single parent or other (mainly single) households), employment status (employed, unemployed or non-working) and years of residence at the address. Annual data on standardised household income ( $<€ 20000, € 20000-€ 35000$ or $>€ 35000)$, and antidepressant prescriptions (yes or no) based on code N06A in accordance with the Anatomical Therapeutic Chemical Classification system were extracted for the year before matching time.

\section{Statistical analysis}

Due to the matched sampling design, we fitted conditional logistic regressions to assess the associations between suicide and neighbourhood characteristics. ${ }^{24}$ The baseline model (model 1 ) included social fragmentation, deprivation and urbanicity. In the fully adjusted model 2, individuallevel variables were added to examine the effect of neighbourhood characteristics net of individual characteristics. We first examined model 1 and 2 for the total sample. Next, we examined both 
Hagedoor, P., Groenewegen, P.P., Roberts, H., Helbich, M. Is suicide mortality associated with neighbourhood social fragmentation and deprivation? A Dutch register-based case-control study using individualised neighbourhoods. Journal of Epidemiology \& Community Health: 2020, 74(2), p. 197-202

models stratified by sex. Finally, we examined the fully adjusted model (model 2) per age group (1839 years and $40-64$ years) for the total sample as well as stratified by sex. Results are presented for the $300 \mathrm{~m}$ buffer as this buffer size had the lowest Akaike information criterion (AIC) scores for the baseline model (results not shown).

As a sensitivity and robustness test, we compared model estimates across different spatial scales by re-fitting model 2 using 600 and $1000 \mathrm{~m}$ buffers as well as administrative neighbourhoods. Model fits were assessed through the AIC. Smaller AIC scores refer to a better fit. In addition, we re-fitted the fully adjusted model (model 2) stratified by length of residence at the current address, differentiating between short-term ( $<5$ years) and long-term ( $>10$ years) residence, to assess associations with neighbourhood characteristics by exposure time. Analyses were performed in Stata (V.14).

\section{Results}

The distribution of cases and controls are shown in table 1 . Of the 9911 suicide cases, $69.3 \%$ were males and $30.7 \%$ were females. Suicide cases were more likely to live in neighbourhoods with high fragmentation and deprivation.

Table 1 shows the regression results for the association between neighbourhood characteristics and suicide mortality before and after adjusting for individual characteristics. The baseline model (model 1) indicated a significantly higher risk of suicide in neighbourhoods with increasing levels of social fragmentation and deprivation in the total population as well as for men and women separately. Suicide risk decreased with increasing urbanicity for the total population and for men, but not for women. The fully adjusted models (model 2) showed that the association between neighbourhood characteristics and suicide were largely attenuated by individual characteristics. The risk of suicide among women remained significantly higher in neighbourhoods with high social fragmentation (OR 1.20; 95\% $\mathrm{Cl} 1.02$ to 1.41). After adjusting for population composition, the risk of suicide was lower in highly deprived neighbourhoods in the total population (OR $0.89 ; 95 \% \mathrm{Cl} 0.82$ to 0.97) and among residents of urbanised neighbourhoods.

\section{[Table 1]}

Fully adjusted analyses stratified by age group, shown in table 2 , indicated associations with social fragmentation among those aged 18-39 years, with deprivation among those aged 40-64 years and with urbanicity among both age groups. Further stratification by sex showed a lower suicide risk among men aged 18-39 years in highly fragmented neighbourhoods and among men aged 40-64 years in highly deprived neighbourhoods. In addition, male suicide risk in both age groups was lower in urbanised neighbourhoods. Associations with social fragmentation and urbanicity among women were only observed for those aged 40-64 years. Associations between neighbourhood characteristics and suicide mortality were comparable across buffer sizes and administrative neighbourhoods (online supplementary table 1), although the strength of the association varied slightly. The stratification of the fully adjusted model (model 2) by years of residence (online supplementary table 2) showed associations with neighbourhood deprivation and social fragmentation in long-term residents ( $>10$ years) and short-term ( $<5$ years) male residents, respectively. 
Hagedoor, P., Groenewegen, P.P., Roberts, H., Helbich, M. Is suicide mortality associated with neighbourhood social fragmentation and deprivation? A Dutch register-based case-control study using individualised neighbourhoods. Journal of Epidemiology \& Community Health: 2020, 74(2), p. 197-202

[Table 2]

\section{Discussion}

\section{Main findings}

The current study examined associations between neighbourhood social fragmentation, deprivation and suicide mortality using individualised neighbourhoods. Unadjusted models showed a significant association between neighbourhood characteristics and suicide mortality. After adjusting for individual characteristics, suicide mortality remained associated with social fragmentation for women, neighbourhood deprivation for the total population and with level of urbanicity for the total population, men and women. Fully adjusted models stratified by age and sex showed a higher suicide risk among women aged 40-64 years in highly fragmented neighbourhoods. We also continued to observe associations with social fragmentation and deprivation for men aged 18-39 years and 40-64 years, respectively. Sensitivity analyses showed that associations with neighbourhood characteristics varied by length of residence, while we observed limited evidence of differences by buffer size.

\section{Interpretation of the findings}

In line with previous studies, ${ }^{5}$ accounting for individual characteristics largely attenuated the association between suicide mortality and neighbourhood characteristics. This indicates that the ecological associations between neighbourhood characteristics and suicide risk mainly reflect underlying compositional differences in individual characteristics between neighbourhoods. Models adjusted for individual characteristics (table 1 ) showed that suicide mortality was associated with marital status, household type, employment status, income and antidepressant use. Compared with these individual-level associations, associations between suicide and neighbourhood characteristics were relatively small in magnitude. However, even after adjusting for population composition, we continued to observe associations with neighbourhood social fragmentation and deprivation.

While for men, adjusting for population composition fully explained the association with social fragmentation, suicide mortality among women remained significantly higher in fragmented neighbourhoods. This is in line with previous studies on suicide, ${ }^{21}$ depression ${ }^{27}$ and mental health. ${ }^{28}$ Women in fragmented neighbourhoods might experience lower levels of social support and increasing levels of neighbourhood disorder and stress. ${ }^{28}$ Older women might be especially reliant on social contacts and social support within the neighbourhood as they spend more time at home, ${ }^{28}$ which might explain why age-stratified models only showed a significant association with social fragmentation for women aged 40-64 years. Compared with the neighbourhood, other social networks such as work or school might be more important sources of social contacts for men and younger age groups, which might act as a buffer against adverse social conditions in the neighbourhood. 2930

Similarly to other European studies, ${ }^{5-10}$ the higher risk of suicide in deprived areas could be explained by differences in population composition. Fully adjusted models stratified by sex and age showed few associations with neighbourhood deprivation, except for a negative association among men aged 40-64 years. This finding is in line with that of other European studies observing a decreased suicide risk among men aged 41-60 years in neighbourhoods with high unemployment ${ }^{8}$ and a higher suicide risk in affluent neighbourhoods. ${ }^{31}$ Although we cannot entirely rule out the possibility of overadjustment due to correlations between (individual-level ${ }^{32}$ ) socioeconomic indicators, this could suggest a potential protective effect of neighbourhood deprivation especially for men. Previous research has shown that suicide among unemployed men was lower in populations with high unemployment rates, ${ }^{33}$ so a context of high deprivation might buffer adverse effects of individual deprivation. In line with previous research, ${ }^{16}$ table 2 showed more pronounced 
Hagedoor, P., Groenewegen, P.P., Roberts, H., Helbich, M. Is suicide mortality associated with neighbourhood social fragmentation and deprivation? A Dutch register-based case-control study using individualised neighbourhoods. Journal of Epidemiology \& Community Health: 2020, 74(2), p. 197-202

associations with individual-level socioeconomic characteristics and suicide among men, which might explain why the negative association with neighbourhood deprivation was only observed among men. In addition, social relationships and feelings of neighbourhood identification, found to be beneficial for mental health, ${ }^{34} 35$ might be stronger in deprived neighbourhoods. ${ }^{34}$ This might explain why especially long-term residence in deprived neighbourhoods results in a reduced suicide risk.

Previous studies in the Netherlands found non-significant correlations between suicide and the level of urbanicity. ${ }^{736}$ However, both studies did not account for differences in individual (socioeconomic) characteristics. Our study showed a decrease in suicide risk in more urbanised neighbourhoods. This is congruent with an ecological German study observing a higher suicide risk in rural areas. ${ }^{23}$ Besides differences in cultural and social norms, rural neighbourhoods might have increased social and geographical isolation as well as less access to (mental) healthcare. ${ }^{22}$ Except for social fragmentation, which showed a stronger association with male suicide mortality in rural areas, we observed no interactions between neighbourhood characteristics and urbanicity on suicide mortality (results not shown).

\section{Strengths and limitations}

A key strength of our study was the use of longitudinal nationwide register data with almost perfect coverage of the entire Dutch population. As the quality of cause of death coding for suicide was evaluated as high in the Netherlands, ${ }^{37}$ our study is likely to cover virtually all suicide deaths during the study period. By combining individual-level indicators with geocoded addresses, we constructed individualised neighbourhoods. This allowed us to explore contextual effects in a more precise way than done so far while circumventing methodological issues related to administrative units. ${ }^{13}$ To our knowledge, this is the first study assessing neighbourhood socioeconomic and social characteristics and suicide using individualised buffers. The case-control design allowed us to compare all suicides with a matched sample of representative controls which resulted in robust estimates while substantially reducing computational intensity. ${ }^{38}$ By matching cases and controls on calendar time and selecting individuals and neighbourhood characteristics at time of suicide, we accounted for changes in attributes during the follow-up period.

Although we included several neighbourhood-level and individual-level variables, data availability was limited to the population register. The construction of the neighbourhood socioeconomic index was therefore restricted by the available socioeconomic indicators. Other potentially relevant neighbourhood characteristics, including social capital and religion found elsewhere to be correlated with suicide, ${ }^{736}$ were unavailable at a detailed spatial scale. As findings might be sensitive to the choice and categorisation of neighbourhood characteristics, we ran sensitivity tests using different categorisations of neighbourhood characteristics (tertiles/quartiles/quintiles) and modelling each indicator separately. Results were similar, indicating robust findings (results not shown). We adjusted for multiple individual-level indicators to explore the independent association between neighbourhood characteristics and suicide. However, we cannot entirely rule out the possibility of overadjustment due to correlations between individual-level (socioeconomic) characteristics ${ }^{32}$ or between individual and neighbourhood characteristics. ${ }^{39}$ We controlled for antidepressant prescriptions as proxy for diagnosed depression. This excluded non-diagnosed persons and depressive patients receiving other kinds of treatment. Moreover, prescriptions do not provide information on reasons for prescription, dose and actual use of medication. It was not possible to conduct a full cohort study as computing the annual social context across the study period for each address (approximately 9 million in 2016) would be computationally too demanding. However, our nested case-control approach provides results that are very similar to full cohort analysis at substantially reduced computation time. ${ }^{38}$ The sample selection was based on the noninstitutionalised population with a 10 -year residential history in the Netherlands, which might have resulted in an under-representation of the immigrant population with a lower socioeconomic 
Hagedoor, P., Groenewegen, P.P., Roberts, H., Helbich, M. Is suicide mortality associated with neighbourhood social fragmentation and deprivation? A Dutch register-based case-control study using individualised neighbourhoods. Journal of Epidemiology \& Community Health: 2020, 74(2), p. 197-202

position. However, these selection criteria were necessary to ensure that the study population was exposed to residential environments for a sufficient amount of time and to allow for stratification by length of residence. As the selection criteria were applied in the selection of both cases and controls, we expect this to have little effects on the results. Finally, although efforts were made to match the social context to time of suicide, our study did not take the social context over past residential locations into account. Yet, our findings by length of residence indicated that associations with neighbourhood characteristics became stronger over time. Our future research will assess environmental exposures of people's residential history further. ${ }^{13}$

\section{Conclusion}

Our findings suggest that next to individual characteristics, the social and economic context within which people live may both enhance and buffer the risk of suicide. Furthermore, associations between suicide and neighbourhood deprivation and fragmentation varied by sex and age group. Next to targeting at-risk individuals, interventions focused on suicide prevention should be targeting at-risk populations including rural inhabitants and women in highly fragmented neighbourhoods. However, to construct effective place-based interventions more research is needed into underlying mechanisms in order to identify specific neighbourhood factors that exacerbate or protect against suicide risk.

\section{What is already known on this subject}

- Most ecological studies reported higher suicide risk in deprived and fragmented neighbourhoods.

- Most European multilevel studies showed no associations between neighbourhood socioeconomic characteristics and suicide mortality.

- Research on social fragmentation and deprivation based on administrative units at one point in time (ie, at baseline) is prone to methodological limitations.

\section{What this study adds}

- Using longitudinal register data, this is the first study assessing associations between neighbourhood fragmentation, deprivation and suicide using annual address-based individualised buffers.

- Associations between neighbourhood deprivation and fragmentation were attenuated after adjusting for individual characteristics.

- Fully adjusted models showed a higher suicide risk among women and a lower suicide risk among men aged 18-39 years in highly fragmented neighbourhoods.

- Suicide risk was lower among men aged 40-64 years in highly deprived neighbourhoods.

- Associations between suicide risk and neighbourhood fragmentation and deprivation were comparable across buffer sizes but varied by years of residence.

Twitter Hannah Roberts @hannah_eroberts and Marco Helbich @HelbichMarco

Acknowledgements The authors would like to thank Statistics Netherlands (CBS) for granting access to their data. The authors would also like to thank the anonymous reviewers for their constructive and insightful comments.

Contributors This study design was developed by $\mathrm{PH}$ and $\mathrm{MH}$. $\mathrm{PH}$ did the data preparation, linkage and statistical analysis. $\mathrm{MH}$ processed the buffers. $\mathrm{PH}$ and $\mathrm{MH}$ had full access to the register data. With contributions from PPG, HR and MH, PH led on interpreting the results and writing the manuscript. The text was edited and the final version of the manuscript was approved by all authors. 
Hagedoor, P., Groenewegen, P.P., Roberts, H., Helbich, M. Is suicide mortality associated with neighbourhood social fragmentation and deprivation? A Dutch register-based case-control study using individualised neighbourhoods. Journal of Epidemiology \& Community Health: 2020, 74(2), p. 197-202

Funding This project received funding from the European Research Council (ERC) under the European Union's Horizon 2020 research and innovation programme (grant agreement no. 714993).

Disclaimer The ERC had no involvement in the study design, writing of the manuscript nor the decision to submit the paper for publication.

Competing interests None declared.

Patient consent for publication Not required.

Ethics approval Ethical approval (FETC17-060) for the NEEDS study (Dynamic Urban Environmental Exposures on Depression and Suicide) was obtained by the Ethics Review Board of Utrecht University.

Provenance and peer review Not commissioned; externally peer reviewed.

Data availability statement Data may be obtained from a third party and are not publicly available. Results are based on own calculations by the authors using non-public microdata from Statistics Netherlands. Under certain conditions, these microdata are accessible for statistical and scientific research.

\section{ORCID iD}

Paulien Hagedoorn http://orcid. org/ 0000- 0001- 5566- 0610

\section{References}

1 WHO. Preventing suicide: A global imperative. Geneva: World Health Organization, 2014.

2 Diez Roux AV, Mair C. Neighborhoods and health. Ann N Y Acad Sci 2010;1186:125-45.

3 Platt S. Inequalities and Suicidal Behavior. In: The International Handbook of Suicide Prevention. Chichester, UK: John Wiley \& Sons, Ltd, 2016: 258-83.

4 Rehkopf DH, Buka SL. The association between suicide and the socio-economic characteristics of geographical areas: a systematic review. Psychol Med 2006;36:145-57.

5 Cairns J-M, Graham E, Bambra C. Area-level socioeconomic disadvantage and suicidal behaviour in Europe: a systematic review. Soc Sci Med 2017;192:102-11.

6 Martikainen P, Blomgren J. The effects of area and individual social characteristics on suicide risk: a multilevel study of relative contribution and effect modification. Eur $\mathrm{J}$ Population 2004;20:323-50.

7 Kunst $A E$, van Hooijdonk $C$, Droomers $M$, et al. Community social capital and suicide mortality in the Netherlands: a cross-sectional registry-based study. BMC Public Health 2013;13:969.

8 Agerbo E, Sterne JAC, Gunnell DJ. Combining individual and ecological data to determine compositional and contextual socio-economic risk factors for suicide. Soc Sci Med 2007;64:45161.

9 O'Reilly D, Rosato M, Connolly S, et al. Area factors and suicide: 5-year follow-up of the Northern Ireland population. Br J Psychiatry 2008;192:106-11.

10 Borrell Cet al. Role of individual and contextual effects in injury mortality: new evidence from small area analysis. Inj Prev 2002;8:297-302.

11 Halonen JI, Vahtera J, Oksanen T, et al. Socioeconomic characteristics of residential areas and risk of death: is variation in spatial units for analysis a source of heterogeneity in observed associations? BMJ Open 2013;3:e002474-9. 
Hagedoor, P., Groenewegen, P.P., Roberts, H., Helbich, M. Is suicide mortality associated with neighbourhood social fragmentation and deprivation? A Dutch register-based case-control study using individualised neighbourhoods. Journal of Epidemiology \& Community Health: 2020, 74(2), p. 197-202

12 Hedman L. The impact of residential mobility on measurements of neighbourhood effects. Housing Studies 2011;26:501-19.

13 Helbich M. Toward dynamic urban environmental exposure assessments in mental health research. Environ Res 2018;161:129-35.

14 Chaix B, Merlo J, Evans D, et al. Neighbourhoods in eco-epidemiologic research: delimiting personal exposure areas. A response to Riva, Gauvin, Apparicio and Brodeur. Soc Sci Med 2009;69:1306-10.

15 OECD. Suicide rates per 100000 persons, 2007-2016, 2019. Available: https:// data. oecd. org/ healthstat/ suicide- rates. htm [Accessed 12 Aug 2019].

16 Li Z, Page A, Martin G, et al. Attributable risk of psychiatric and socio-economic factors for suicide from individual-level, population-based studies: a systematic review. Soc Sci Med 2011;72:60816.

17 Helbich M. Dynamic Urban Environmental Exposures on Depression and Suicide (NEEDS) in the Netherlands: a protocol for a cross-sectional smartphone tracking study and a longitudinal population register study. BMJ Open 2019;9:e030075.

18 Bakker BFM, Van Rooijen J, Van Toor L. The system of social statistical datasets of statistics Netherlands: an integral approach to the production of register-based social statistics. Stat J IAOS 2014;30:411-24.

19 Vandenbroucke JP, Pearce N. Case-Control studies: basic concepts. Int J Epidemiol 2012;41:1480-9.

20 Kruger DJ, Reischl TM, Gee GC. Neighborhood social conditions mediate the association between physical deterioration and mental health. Am J Community Psychol 2007;40:261-71.

21 Congdon P. Assessing the impact of socioeconomic variables on small area variations in suicide outcomes in England. Int J Environ Res Public Health 2013;10:158-77.

22 Hirsch JK, Cukrowicz KC. Suicide in rural areas: an updated review of the literature. Journal of Rural Mental Health 2014;38:65-78.

23 Helbich M, Blüml V, de Jong $\mathrm{T}$, et al. Urban-rural inequalities in suicide mortality: a comparison of urbanicity indicators. Int J Health Geogr 2017;16:39.

24 Keogh RH, Cox DR. Matched case-control studies. In: Case-Control Studies. Cambridge: Cambridge University Press, 2014: 62-82.

25 Batty GD, Kivimäki M, Bell S, et al. Psychosocial characteristics as potential predictors of suicide in adults: an overview of the evidence with new results from prospective cohort studies. Transl Psychiatry 2018;8:22.

26 Foster T. Adverse life events proximal to adult suicide: a synthesis of findings from psychological autopsy studies. Arch Suicide Res 2011;15:1-15.

27 Bagheri N, Batterham PJ, Salvador-Carulla L, et al. Development of the Australian neighborhood social fragmentation index and its association with spatial variation in depression across communities. Soc Psychiatry Psychiatr Epidemiol 2019;54:1189-98.

28 Ivory VC, Collings SC, Blakely T, et al. When does neighbourhood matter? Multilevel relationships between neighbourhood social fragmentation and mental health. Soc Sci Med 2011;72:1993-2002.

29 Hoogerbrugge MM, Burger MJ. Neighborhood-Based social capital and life satisfaction: the case of Rotterdam, the Netherlands. Urban Geogr 2018;39:1484-509.

30 Fagg J, Curtis S, Stansfeld SA, et al. Area social fragmentation, social support for individuals and psychosocial health in young adults: evidence from a national survey in England. Soc Sci Med 2008;66:242-54.

31 Panczak R, Galobardes B, Voorpostel M, et al. A Swiss neighbourhood index of socioeconomic position: development and association with mortality. J Epidemiol Community Health 2012;66:1129-36. 
Hagedoor, P., Groenewegen, P.P., Roberts, H., Helbich, M. Is suicide mortality associated with neighbourhood social fragmentation and deprivation? A Dutch register-based case-control study using individualised neighbourhoods. Journal of Epidemiology \& Community Health: 2020, 74(2), p. 197-202

32 Galobardes B, Shaw M, Lawlor DA, et al. Indicators of socioeconomic position (Part 1). J Epidemiol Community Health 2006;60:7-12.

33 Crawford MJ, Kuforiji B, Ghosh P. The impact of social context on socio-demographic risk factors for suicide: a synthesis of data from case-control studies. J Epidemiol Community Health 2010;64:530-4.

34 Kim J. Neighborhood disadvantage and mental health: the role of neighbourhood disorder and social relationships. Soc Sci Res 2010;39:260-71.

35 Fong P, Cruwys T, Haslam C, et al. Neighbourhood identification and mental health: how social identification moderates the relationship between socioeconomic disadvantage and health. $J$ Environ Psychol 2019;61:101-14.

36 Helbich M, de Beurs D, Kwan M-P, et al. Natural environments and suicide mortality in the Netherlands: a cross-sectional, ecological study. The Lancet Planetary Health 2018;2:e134-9.

37 Harteloh P, de Bruin K, Kardaun J. The reliability of cause-of- death coding in the Netherlands. Eur J Epidemiol 2010;25:531-8.

38 Essebag V, Platt RW, Abrahamowicz M, et al. Comparison of nested case-control and survival analysis methodologies for analysis of time-dependent exposure. BMC Med Res Methodol 2005;5:1-6.

39 Diez-Roux AV. Bringing context back into epidemiology: variables and fallacies in multilevel analysis. Am J Public Health 1998;88:216-22. 
Hagedoor, P., Groenewegen, P.P., Roberts, H., Helbich, M. Is suicide mortality associated with neighbourhood social fragmentation and deprivation? A Dutch register-based case-control study using individualised neighbourhoods. Journal of Epidemiology \& Community Health: 2020, 74(2), p. 197-202

\section{Tables}

Table 1 ORs (95\% Cls) for suicide mortality by neighbourhood and individual characteristics $(300 \mathrm{~m}$ buffer), total population, men and women aged 18-64 years

\begin{tabular}{|c|c|c|c|c|c|c|c|c|}
\hline & \multicolumn{2}{|c|}{ Distribution (\%) } & \multicolumn{2}{|l|}{ Total } & \multicolumn{2}{|l|}{ Men } & \multicolumn{2}{|l|}{ Women } \\
\hline & Cases & Controls & Model 1 & Model 2 & Model 1 & Model 2 & Model 1 & Model 2 \\
\hline \multicolumn{9}{|l|}{ Neighbourhood characteristics } \\
\hline \multicolumn{9}{|l|}{ Social fragmentation } \\
\hline Q1 (low) (ref.) & 19.8 & 25.5 & 1.00 & 1.00 & 1.00 & 1.00 & 1.00 & 1.00 \\
\hline Q2 & 22.9 & 25.2 & 1.12 (1.05 to 1.19$)$ & 1.03 (0.96 to 1.11$)$ & 1.12 (1.04 to 1.21$)$ & 1.03 (0.94 to 1.12 ) & 1.11 (0.98 to 1.26$)$ & 1.03 (0.89 to 1.19$)$ \\
\hline Q3 & 25.5 & 25.0 & 1.19 (1.11 to 1.28$)$ & 0.99 (0.92 to 1.07$)$ & 1.14 (1.05 to 1.24$)$ & 0.95 (0.87 to 1.04$)$ & $1.32(1.17$ to 1.50$)$ & 1.10 (0.95 to 1.27$)$ \\
\hline Q4 (high) & 31.8 & 24.3 & 1.52 (1.41 to 1.63$)$ & 0.99 (0.90 to 1.08$)$ & $1.43(1.31$ to 1.57$)$ & 0.91 (0.82 to 1.01$)$ & $1.72(1.50$ to 1.97$)$ & $1.20(1.02$ to 1.41$)$ \\
\hline \multicolumn{9}{|l|}{ Socioeconomic deprivation } \\
\hline Q1 (low) (ref.) & 20.2 & 25.5 & 1.00 & 1.00 & 1.00 & 1.00 & 1.00 & 1.00 \\
\hline Q2 & 22.2 & 25.3 & 1.06 (1.00 to 1.14$)$ & 0.95 (0.89 to 1.02$)$ & 1.06 (0.98 to 1.14$)$ & 0.95 (0.87 to 1.03$)$ & 1.08 (0.96 to 1.22$)$ & 0.97 (0.85 to 1.12 ) \\
\hline Q3 & 27.0 & 24.8 & 1.25 (1.17 to 1.33$)$ & $1.02(0.95$ to 1.10$)$ & 1.26 (1.16 to 1.36$)$ & 1.04 (0.95 to 1.13$)$ & 1.22 (1.08 to 1.38$)$ & 0.98 (0.85 to 1.13 ) \\
\hline Q4 (high) & 30.6 & 24.4 & 1.32 (1.23 to 1.42$)$ & 0.89 (0.82 to 0.97$)$ & 1.33 (1.22 to 1.44$)$ & 0.91 (0.83 to 1.00$)$ & 1.30 (1.15 to 1.48$)$ & 0.86 (0.74 to 1.00$)$ \\
\hline \multicolumn{9}{|l|}{ Urbanicity } \\
\hline Q1 (low) (ref.) & 23.0 & 25.2 & 1.00 & 1.00 & 1.00 & 1.00 & 1.00 & 1.00 \\
\hline Q2 & 24.8 & 25.0 & 1.01 (0.95 to 1.07 ) & 0.93 (0.87 to 0.99$)$ & 0.99 (0.92 to 1.07 ) & 0.95 (0.88 to 1.03$)$ & 1.05 (0.93 to 1.17$)$ & 0.86 (0.75 to 0.98 ) \\
\hline Q3 & 25.0 & 25.0 & 0.97 (0.91 to 1.03$)$ & 0.87 (0.81 to 0.93$)$ & 0.95 (0.88 to 1.02$)$ & 0.89 (0.82 to 0.97$)$ & 1.02 (0.91 to 1.14$)$ & 0.81 (0.71 to 0.93 ) \\
\hline Q4 (high) & 27.3 & 24.8 & 0.91 (0.86 to 0.98 ) & 0.80 (0.74 to 0.86$)$ & 0.90 (0.84 to 0.98 ) & 0.82 (0.75 to 0.90$)$ & 0.94 (0.83 to 1.06$)$ & 0.74 (0.64 to 0.85 ) \\
\hline \multicolumn{9}{|l|}{ Individual characteristics } \\
\hline \multicolumn{9}{|l|}{ Ethnic origin } \\
\hline Dutch (ref.) & 85.7 & 84.6 & & 1.00 & & 1.00 & & 1.00 \\
\hline Non-Dutch & 14.3 & 15.5 & & $0.78(0.72$ to 0.83$)$ & & 0.76 (0.7 to 0.82$)$ & & 0.83 (0.73 to 0.94$)$ \\
\hline \multicolumn{9}{|l|}{ Marital status } \\
\hline Married (ref.) & 35.6 & 59.5 & & 1.00 & & 1.00 & & 1.00 \\
\hline Never married & 41.7 & 29.1 & & 1.35 (1.25 to 1.46$)$ & & 1.31 (1.19 to 1.43$)$ & & $1.47(1.26$ to 1.70$)$ \\
\hline Non-married & 22.7 & 11.4 & & 1.43 (1.32 to 1.55$)$ & & 1.43 (1.30 to 1.58$)$ & & 1.44 (1.24 to 1.68$)$ \\
\hline \multicolumn{9}{|l|}{ Household type } \\
\hline Couple with kids (ref.) & 28.6 & 49.4 & & 1.00 & & 1.00 & & 1.00 \\
\hline Couple without kids & 20.2 & 29.3 & & 1.13 (1.06 to 1.21$)$ & & 0.99 (0.91 to 1.07$)$ & & 1.59 (1.40 to 1.81$)$ \\
\hline Single parent & 7.7 & 5.5 & & 1.69 (1.52 to 1.88$)$ & & 1.77 (1.55 to 2.01$)$ & & 1.76 (1.46 to 2.13 ) \\
\hline Other & 43.5 & 15.9 & & 3.07 (2.84 to 3.31 ) & & 2.81 (2.57 to 3.07 ) & & 4.11 (3.50 to 4.82 ) \\
\hline \multicolumn{9}{|l|}{ Employment status } \\
\hline Employed (ref.) & 43.1 & 74.9 & & 1.00 & & 1.00 & & 1.00 \\
\hline Unemployed & 3.6 & 2.5 & & 2.26 (1.99 to 2.57 ) & & 2.37 (2.05 to 2.73$)$ & & 1.92 (1.45 to 2.54$)$ \\
\hline Non-working & 53.4 & 22.6 & & 3.70 (3.49 to 3.91 ) & & 3.79 (3.53 to 4.05$)$ & & 3.68 (3.32 to 4.07$)$ \\
\hline \multicolumn{9}{|l|}{ Income } \\
\hline Low (<€20 000) (ref.) & 43.1 & 26.8 & & 1.00 & & 1.00 & & 1.00 \\
\hline Medium ( $€ 20000-€ 35000)$ & 42.5 & 50.8 & & 1.01 (0.96 to 1.07$)$ & & 0.99 (0.93 to 1.06$)$ & & 1.07 (0.96 to 1.19$)$ \\
\hline High $(>€ 35000)$ & 14.5 & 22.4 & & 0.99 (0.91 to 1.07$)$ & & 0.89 (0.81 to 0.98$)$ & & 1.34 (1.16 to 1.56$)$ \\
\hline \multicolumn{9}{|l|}{ Antidepressant prescription } \\
\hline No (ref.) & 62.2 & 93.4 & & 1.00 & & 1.00 & & 1.00 \\
\hline Yes & 37.8 & 6.6 & & 6.98 (6.60 to 7.38$)$ & & $6.25(5.82$ to 6.71$)$ & & 8.49 (7.73 to 9.32$)$ \\
\hline
\end{tabular}

Model 1: adjusted for all neighbourhood characteristics.

Model 2: adjusted for all neighbourhood and individual characteristics.

ref., reference category. 
Hagedoor, P., Groenewegen, P.P., Roberts, H., Helbich, M. Is suicide mortality associated with neighbourhood social fragmentation and deprivation? A Dutch register-based case-control study using individualised neighbourhoods. Journal of Epidemiology \& Community Health: 2020, 74(2), p. 197-202

Table 2 ORs (95\% Cls) for suicide mortality by neighbourhood characteristics* (300 $\mathrm{m}$ buffer) and age group, total sample, men and women

\begin{tabular}{|c|c|c|c|c|c|c|}
\hline & \multicolumn{2}{|l|}{ Total } & \multicolumn{2}{|l|}{ Men } & \multicolumn{2}{|l|}{ Women } \\
\hline & $18-39$ years & $40-64$ years & $18-39$ years & $40-64$ years & $18-39$ years & $40-64$ years \\
\hline \multicolumn{7}{|c|}{ Neighbourhood characteristics } \\
\hline \multicolumn{7}{|c|}{ Social fragmentation } \\
\hline Q1 (low) (ref.) & 1.00 & 1.00 & 1.00 & 1.00 & 1.00 & 1.00 \\
\hline Q2 & 0.98 (0.84 to 1.14$)$ & 1.05 (0.96 to 1.14$)$ & 0.98 (0.82 to 1.17$)$ & 1.05 (0.95 to 1.15$)$ & 0.93 (0.68 to 1.28$)$ & 1.05 (0.90 to 1.23$)$ \\
\hline Q3 & 0.97 (0.83 to 1.14$)$ & 1.00 (0.91 to 1.09 ) & 0.98 (0.81 to 1.18$)$ & 0.95 (0.85 to 1.05 ) & 0.95 (0.69 to 1.31 ) & 1.14 (0.97 to 1.35$)$ \\
\hline Q4 (high) & 0.82 (0.69 to 0.98$)$ & 1.08 (0.98 to 1.19$)$ & 0.78 (0.64 to 0.96$)$ & 0.99 (0.88 to 1.12$)$ & 0.93 (0.66 to 1.32 ) & 1.32 (1.09 to 1.59$)$ \\
\hline \multicolumn{7}{|c|}{ Socioeconomic deprivation } \\
\hline Q1 (low) (ref.) & 1.00 & 1.00 & 1.00 & 1.00 & 1.00 & 1.00 \\
\hline Q2 & 0.99 (0.85 to 1.14$)$ & 0.94 (0.87 to 1.02 ) & 1.00 (0.84 to 1.20$)$ & 0.93 (0.85 to 1.03 ) & 0.94 (0.71 to 1.26$)$ & 0.97 (0.83 to 1.14$)$ \\
\hline Q3 & $1.16(1.00$ to 1.35$)$ & 0.97 (0.89 to 1.06$)$ & 1.28 (1.07 to 1.52$)$ & 0.97 (0.87 to 1.07$)$ & 0.91 (0.67 to 1.23$)$ & 1.00 (0.85 to 1.17$)$ \\
\hline Q4 (high) & 1.04 (0.88 to 1.22$)$ & 0.84 (0.76 to 0.92 ) & 1.13 (0.94 to 1.37$)$ & 0.84 (0.75 to 0.94$)$ & 0.85 (0.62 to 1.16$)$ & 0.85 (0.71 to 1.01$)$ \\
\hline \multicolumn{7}{|l|}{ Urbanicity } \\
\hline Q1 (low) (ref.) & 1.00 & 1.00 & 1.00 & 1.00 & 1.00 & 1.00 \\
\hline Q2 & 0.91 (0.79 to 1.05 ) & 0.93 (0.86 to 1.00$)$ & 0.94 (0.8 to 1.11$)$ & 0.95 (0.87 to 1.04 ) & 0.83 (0.62 to 1.12 ) & 0.87 (0.75 to 1.00$)$ \\
\hline Q3 & 0.84 ( 0.73 to 0.97$)$ & 0.88 (0.81 to 0.95$)$ & 0.84 ( 0.71 to 0.99 ) & 0.90 (0.82 to 0.99 ) & 0.85 (0.64 to 1.13$)$ & 0.81 ( 0.70 to 0.94$)$ \\
\hline Q4 (high) & 0.76 (0.66 to 0.88 ) & 0.81 (0.75 to 0.89 ) & 0.71 (0.60 to 0.85$)$ & 0.87 (0.78 to 0.97 ) & 0.89 (0.66 to 1.20$)$ & 0.70 (0.59 to 0.82$)$ \\
\hline
\end{tabular}

* Results based on model 2: adjusted for all neighbourhood and individual characteristics. ref., reference category. 